\title{
Point X-Ray Sources in the Supernova Remnant RCW 86
}

\author{
Vasilii V. Gvaramadze \\ Sternberg Astronomical Institute, Moscow State University, \\ Universitetskij Pr. 13, Moscow, 119992, Russia
}

\author{
Alexey A. Vikhlinin \\ Space Research Institute, Russian Academy of Sciences, \\ Profsoyuznaya 84/32, Moscow, 117997, Russia
}

\begin{abstract}
We have discovered two point X-ray sources in the southwest protrusion of the supernova remnant (SNR) RCW 86. One of them is interpreted as a foreground active star, while the second one as a candidate neutron star associated with the SNR.
\end{abstract}

\section{Introduction}

We report the results of search for a stellar remnant in the southwest protrusion of the SNR RCW 86 (G 315.4-2.30, MSH 14-63) using the archival ACIS data of the Chandra X-ray Observatory. The search was motivated by a hypothesis that RCW 86 is the result of an off-centered cavity supernova (SN) explosion of a moving massive star, which ends its evolution just near the edge of the main-sequence wind-driven bubble (Gvaramadze 2002). This hypothesis implies that the southwest protrusion in RCW 86 is the remains of a preexisting bow shock-like structure created by the interaction of the SN progenitor's wind with the interstellar medium, and that the actual location of the SN blast center is near the center of this hemispherical structure. We have discovered two point X-ray sources in the "proper" place (for details see Gvaramadze \& Vikhlinin 2003).

\section{Point X-ray sources}

Fig. 1 shows the Chandra $(0.7-2 \mathrm{keV})$ image of two point X-ray sources at $\alpha_{2000}=$ $14^{\mathrm{h}} 40^{\mathrm{m}} 31^{\mathrm{s}} .33, \delta_{2000}=-62^{\circ} 38^{\prime} 22^{\prime \prime} .8$ (labelled as $\mathrm{S}$ ) and $\alpha_{2000}=14^{\mathrm{h}} 40^{\mathrm{m}} 31^{\mathrm{s}} .05, \delta_{2000}$ $=-62^{\circ} 38^{\prime} 16^{\prime \prime} .9$ (labelled as $\left.\mathrm{N}\right)$.

The source $\mathrm{S}$ has an optical counterpart with the photographic magnitude $13.38 \pm 0.40$. The spectrum of this source can be fitted with an optically thin plasma model with depleted abundance. We interpret this source as a foreground active star of late spectral type.

The source $\mathrm{N}$ has no optical counterpart to a limiting magnitude $\sim 21$. The spectrum of the source (shown in Fig. 2) can be fitted equally well with several simple models (power law: photon index $=1.87$; two-temperature blackbody: 


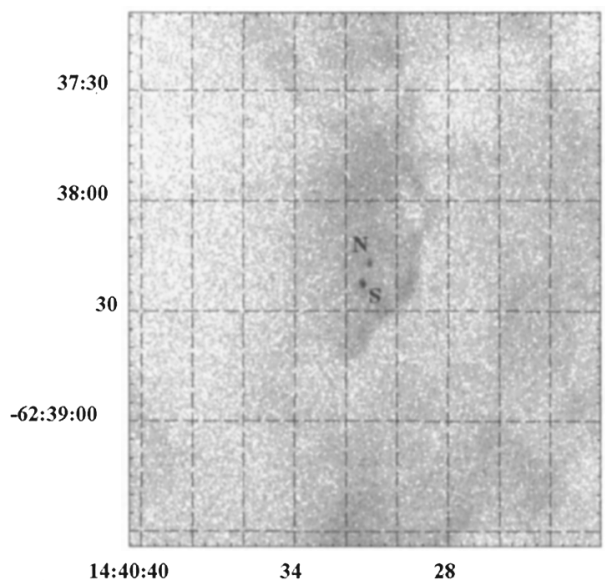

Figure 1. The Chandra image of point X-ray sources in RCW 86.

$k T_{1}=0.11 \mathrm{keV}, R_{1}=2.34 \mathrm{~km}$ and $k T_{2}=0.71 \mathrm{keV}, R_{2}=0.06 \mathrm{~km}$; blackbody plus power law: $k T=0.07 \mathrm{keV}$, photon index $=2.3$ ). We interpret this source as a candidate neutron star, while the photon index and nonthermal X-ray luminosity $\left(\sim 10^{32} \mathrm{erg} \mathrm{s}^{-1}\right)$ of the source (almost the same as those of the Vela pulsar and the recently discovered pulsar PSR J 0205+6449 in the SNR 3C 58) suggest that it can be a young "ordinary" pulsar.

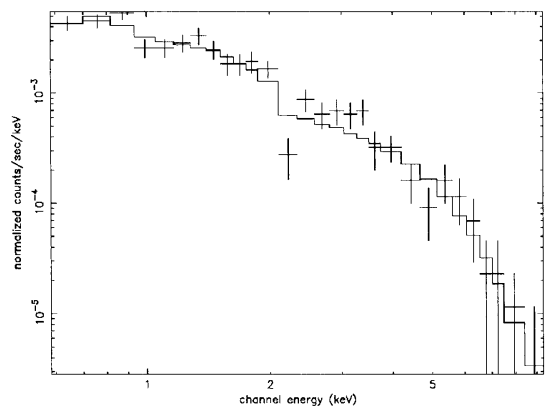

Figure 2. The background-subtracted Chandra ACIS spectrum from the source N. The solid line corresponds to the best fit blackbody plus power law model.

\section{References}

Gvaramadze, V.V. 2002, in New Visions of the X-ray Universe in the XMMNewton and Chandra Era, ed. F. Jansen (ESA SP-488), in press (astro$\mathrm{ph} / 0208028)$

Gvaramadze, V.V., \& Vikhlinin, A.A. 2003, A\&A, in press (astro-ph/0208030) 